\title{
Assessing the Impact of Vaccination on Controlling the Spread of Human Scabies
}

\author{
C. P. Bhunu, ${ }^{1}$ S. Mushayabasa, ${ }^{1}$ and T. G. Monera $^{2}$ \\ ${ }^{1}$ Department of Mathematics, University of Zimbabwe, P.O. Box MP 167, Mount Pleasant, Harare, Zimbabwe \\ ${ }^{2}$ School of Pharmacy, University of Zimbabwe, P.O. Box MP 167, Mount Pleasant, Harare, Zimbabwe \\ Correspondence should be addressed to C. P. Bhunu; cpbhunu@gmail.com
}

Received 4 February 2013; Accepted 13 March 2013

Academic Editors: F. Castiglione, G. Colonna, A. Fedorov, S. A. Oprisan, and D. Xu

Copyright (c) 2013 C. P. Bhunu et al. This is an open access article distributed under the Creative Commons Attribution License, which permits unrestricted use, distribution, and reproduction in any medium, provided the original work is properly cited.

\begin{abstract}
Scabies is among the infestations almost forgotten due to its association with poor communities. We formulate a deterministic model to assess the possible impact vaccination will have on scabies control. The Descartes's rule of signs is used to show the nature of the endemic equilibria. Analysis of the reproduction number and numerical simulations suggest that vaccination in addition to treatment will help greatly in reducing the spread of scabies infestation. This suggests there is a strong need for researchers to come up with a possible vaccine in that order to effectively control scabies especially among the disadvantaged communities.
\end{abstract}

\section{Introduction}

Scabies is a highly contagious skin infestation caused by skin infestation with the mite Sarcoptes scabiei and is spread by direct contact. Its common name is the "itch mite" derived from the severe pruritis it causes [1]. The female mite burrows the skin and lays its eggs which hatch into larvae within 2-3 days [2]. The larvae then burrow to reach the skin surface, then dig burrows where they pass through two further developmental stages (protonymphs and tritonymphs) before moulting into either male or female mites. As a result a result, S-shaped trails accompanied by small insect bites cover infested areas. It is only less than $1 \%$ of the eggs laid by female mites that develop into adult mites [3]. Following infection, the mite population increases rapidly up to 25 adult mites after 50 days and upto 500 mites by 100 days [3], and thereafter rapid decrease may be due to scratching as well as host immune's response. However, 10-12 mites are enough to cause a burden in a host [1].

It is estimated that, despite the availability of effective treatment, over 300 million people in the world are scabies infested [6]. Scabies is well described in the historical record with descriptions evident in ancient texts from China, India and the Middle East [7]. Scabies was also a scourge in London during the First World War upto 1920 [8]. Scabies can be treated with any of the following medications: permethrin (one of the most effective and most expensive), ivermectin (an oral medicine shown in clinical studies to be effective against scabies but not recommended for children), lindane (effective though there are concerns that it can cause neurotoxicity), benzyl benzoate, crotamiton, malathion, and sulphur preparations. Due to low cost, benzyl-benzoate and sulphur ointments are commonly used in developing countries. In general the prevalence of scabies is not well documented especially in Africa [2]. Scabies infestations like other infectious diseases are more common among the poor due to the overcrowded and unhygienic living conditions they are exposed to. However, the issues of associating poverty and scabies are complicated as even in the developed world scabies cases have also being recently reported [9]. In November 2007, the Public Health Service in Amsterdam was notified that the nursing home doctor in Amsterdam had been diagnosed with scabies and that another staff member who worked in the same nursing home had been diagnosed with scabies in September 2007 [9].

Currently, there is no vaccine against scabies; however, the observations that scabies infestation in humans is self limiting and that after the first infestation, significant protective immunity against re-infestation provide theoretical support for the development of a vaccine against scabies 
[10-12]. A vaccine against scabies mites would help improve the quality of life of poor people in the world who live in overcrowded and unhygenic conditions. Scabies being a neglected disease associated with the poor to the best knowledge of the authors no mathematical insight into its transmission dynamics have been attempted. This is possibly a first attempt to analyse the potential benefits a vaccine will have on scabies control. This work seeks to address whether the current treatment regime against scabies mites is enough or there is a need to come up with new vaccines.

The rest of this paper is organised as follows. In the next section, the model and its basic properties are presented. In Section 3 , the equilibria states of the model are determined and detailed analysis of the model is carried out. The possible effects the intervention strategies have on scabies control are assessed through numerical simulations, and finally a discussion in the last section presented.

\section{Model Formulation}

The population is divided into four epidemiological classes according to an individual's disease status, namely, the unvaccinated susceptibles $\left(S_{u}\right)$, vaccinated susceptibles $\left(S_{v}\right)$, infected individuals $(I)$, and the recovered $(R)$. Thus, the total human population is given by $N(t)=S_{u}(t)+S_{v}(t)+$ $I(t)+R(t)$. The human population is recruited through birth at a rate $\Lambda$ into the unvaccinated susceptible class. Unvaccinated susceptibles are vaccinated at a rate $\alpha$ to move into the vaccinated susceptible class $\left(S_{v}\right)$. The vaccine wanes at a rate $\rho$ for individuals in $S_{v}$ to move back into $S_{u}$ class. Following prolonged contact and/or sexual contact with scabies infected person, the unvaccinated susceptibles are infected with scabies at a rate $\lambda$ to move into the infected class $(I)$ where $\lambda=\beta I / N$ with $\beta$ being the product of the probability of getting infected per contact with an infected case and the effective contact rate necessary for scabies infestation to occur. Even though currently there is no effective vaccine against scabies, we assume vaccinated susceptibles have a reduced rate of becoming infected; thus, vaccinated susceptibles are infected with scabies at a rate $\delta \lambda$ with $0<\delta<1$ signifying the protective factor of the vaccine. The infected individuals are treated and move into the recovered class $(R)$ at a rate $\gamma$. Recovery following treatment confers some partial immunity against reinfection [13], so individuals in $R(t)$ class are reinfected at a rate $\sigma \lambda, \sigma \in$ $(0,1)$. Individuals in all human subclasses experience natural death at a rate $\mu$. On their own, scabies do not kill people, so there is no disease induced death in this model The model flow diagram is shown in Figure 1.

Based on the assumptions, the following system of differential equations describes the model:

$$
\begin{aligned}
& S_{u}^{\prime}(t)=\Lambda+\rho S_{v}-\lambda S_{u}-(\mu+\alpha) S_{u}, \\
& S_{v}^{\prime}(t)=\alpha S_{u}-\delta \lambda S_{v}-(\mu+\rho) S_{v}, \\
& I^{\prime}(t)=\lambda S_{u}+\delta \lambda S_{v}+\sigma \lambda R-(\mu+\gamma) I, \\
& R^{\prime}(t)=\gamma I-\sigma \lambda R-\mu R .
\end{aligned}
$$

Adding all equations in model system (1), we have

$$
N^{\prime}(t)=\Lambda-\mu N \Longrightarrow N(t)=N(0) e^{-\mu t}+\frac{\Lambda}{\mu}\left(1-e^{-\mu t}\right) .
$$

Thus, $\lim _{t \rightarrow \infty} N(t)=\Lambda / \mu$ which is the limiting value of the population. Therefore without loss of generality, we can only consider solutions of model system (2) in the following positively invariant, that is, all solutions in biological space where all the variables are nonnegative:

$$
\Omega=\left\{\left(S_{u}, S_{v}, I, R\right) \mid N \leq \frac{\Lambda}{\mu}\right\} .
$$

\section{Disease-Free Equilibrium and Stability Analysis}

The disease-free equilibrium of model system (1) is given by

$$
E^{0}=\left(S_{u}^{0}, S_{v}^{0}, I^{0}, R^{0}\right)=\left(\frac{\Lambda(\mu+\rho)}{\mu(\mu+\rho+\alpha)}, \frac{\Lambda \alpha}{\mu(\mu+\rho+\alpha)}, 0,0\right) .
$$

The reproduction number of model system (1) is given by

$$
\mathscr{R}_{P_{T}}=\frac{\beta(\mu+\rho+\delta \alpha)}{(\mu+\gamma)(\mu+\rho+\alpha)} .
$$

Theorem 1 follows from [14, Theorem 2].

Theorem 1. The disease-free equilibrium $\mathscr{E}^{0}$ is locally asymptotically stable for $\mathscr{R}_{P_{T}}<1$ and unstable otherwise.

3.1. Endemic Equilibrium. The endemic equilibrium of model system (1) is given by $E^{*}=\left(S_{u}^{*}, S_{v}^{*}, I^{*}, R^{*}\right)$ with

$$
\begin{aligned}
S_{u}^{*}= & \frac{\Lambda\left(\delta \lambda^{*}+\mu+\rho\right)}{\delta \lambda^{* 2}+(\delta(\mu+\alpha)+\mu+\rho) \lambda^{*}+\mu(\mu+\alpha+\rho)}, \\
S_{v}^{*}= & \frac{\Lambda \alpha}{\delta \lambda^{* 2}+(\delta(\mu+\alpha)+\mu+\rho) \lambda^{*}+\mu(\mu+\alpha+\rho)}, \\
I^{*}= & \left(\Lambda \lambda^{*}\left(\sigma \lambda^{*}+\mu\right)\left(\delta \lambda^{*}+\mu+\rho+\delta \alpha\right)\right) \\
& \times\left(\mu ( \sigma \lambda ^ { * } + \mu + \gamma ) \left[\delta \lambda^{* 2}+(\delta(\mu+\alpha)+\mu+\rho) \lambda^{*}\right.\right. \\
& +\mu(\mu+\alpha+\rho)])^{-1}, \\
I^{*}= & \left(\Lambda \lambda^{*} \gamma\left(\delta \lambda^{*}+\mu+\rho+\delta \alpha\right)\right) \\
& \times\left(\mu ( \sigma \lambda ^ { * } + \mu + \gamma ) \left[\delta \lambda^{* 2}+(\delta(\mu+\alpha)+\mu+\rho) \lambda^{*}\right.\right. \\
& +\mu(\mu+\alpha+\rho)])^{-1}
\end{aligned}
$$

in terms of the force of infection $\lambda^{*}$. Substituting into the equation for the force of infection $\lambda^{*}=\beta I^{*} / N^{*}$ to obtain

$$
\lambda^{*} h\left(\lambda^{*}\right)=\lambda^{*}\left(A \lambda^{* 3}+B \lambda^{* 2}+C \lambda^{*}+D\right)=0,
$$


TABLE 1: Number of possible positive roots of $h\left(\lambda^{*}\right)=0$ for $\mathscr{R}_{P_{T}}>1$ and $\mathscr{R}_{P_{T}}<1$.

\begin{tabular}{|c|c|c|c|c|c|c|c|}
\hline Cases & $A$ & $B$ & $C$ & $D$ & $\mathscr{R}_{P_{T}}$ & Number of sign changes & Number of possible real roots (endemic equilibrium) \\
\hline \multirow[t]{2}{*}{1} & + & + & + & + & $\mathscr{R}_{P_{T}}<1$ & 0 & 0 \\
\hline & + & + & + & - & $\mathscr{R}_{P_{T}}>1$ & 1 & 1 \\
\hline \multirow{2}{*}{2} & + & - & + & + & $\mathscr{R}_{P_{T}}<1$ & 2 & 0,2 \\
\hline & + & - & + & - & $\mathscr{R}_{P_{T}}>1$ & 3 & 1,3 \\
\hline \multirow{2}{*}{3} & + & - & - & + & $\mathscr{R}_{P_{T}}<1$ & 2 & 0,2 \\
\hline & + & - & - & - & $\mathscr{R}_{P_{T}}>1$ & 1 & 1 \\
\hline \multirow{2}{*}{4} & + & + & - & + & $\mathscr{R}_{P_{T}}<1$ & 2 & 0,2 \\
\hline & + & + & - & - & $\mathscr{R}_{P_{T}}>1$ & 1 & 1 \\
\hline
\end{tabular}

TABLE 2: Effects of different levels of vaccination and vaccine waning rates.

\begin{tabular}{lll}
\hline$\alpha<\alpha^{c}$ & $\alpha>\alpha^{c}$ \\
\hline$\rho<\rho^{c}$ & $\begin{array}{l}\text { As much as vaccine waning rates are low, the vaccination } \\
\text { rates are not high enough to control scabies }\end{array}$ & $\begin{array}{l}\text { Ideal situation where vaccination rates are high and vaccine } \\
\text { waning rates are low. In this case, vaccination leads to the } \\
\text { control of scabies. }\end{array}$ \\
\hline$\rho>\rho^{c}$ & $\begin{array}{l}\text { Low vaccination rates with a vaccine which wanes too } \\
\text { quickly cannot control scabies in a community }\end{array}$ & $\begin{array}{l}\text { High vaccination rates with a vaccine which wanes too } \\
\text { quickly have little effect on scabies control }\end{array}$ \\
\hline
\end{tabular}

TABle 3: Model parameters.

\begin{tabular}{lcc}
\hline Model parameter & Value (range) & Source \\
\hline$\Lambda$ & $0.029 \mathrm{yr}^{-1} \times 10000$ & CSOZ \\
$\mu$ & $0.02 \mathrm{yr}^{-1}$ & CSOZ \\
$\beta$ & $0.043 \mathrm{yr}^{-1}$ & Landwehr et al., 1998 [4] \\
$\delta$ & $0.125(0.0-1.0)$ & Assumed \\
$\sigma$ & $0.25(0.0-1.0)$ & Assumed \\
$\alpha$ & $0.8 \mathrm{yr}^{-1}$ & Bhunu et al., 2008 [5] \\
$\rho$ & $0.0002 \mathrm{yr}^{-1}$ & Bhunu et al., 2008 [5] \\
$\gamma$ & $0.75 \mathrm{yr}^{-1}$ & Assumed \\
\hline
\end{tabular}

where $\lambda^{*}=0$ corresponds to the disease-free equilibrium and $h\left(\lambda^{*}\right)=0$ corresponds to the existence of endemic equilibria where

$$
\begin{aligned}
& A=\frac{\sigma \delta}{\mu(\mu+\gamma)(\mu+\alpha+\rho)}, \\
& B=\frac{\delta}{\mu(\mu+\alpha+\rho)}+\frac{\sigma(\mu+\rho+\delta(\mu+\alpha-\beta))}{\mu(\mu+\gamma)(\mu+\alpha+\rho)}, \\
& C=\frac{\mu+\rho+\delta \alpha}{\mu(\mu+\alpha+\rho)}-\frac{\sigma \mathscr{R}_{P_{T}}}{\mu}+\frac{\sigma}{\mu+\gamma}+\frac{\delta(\mu+\gamma-\beta)}{(\mu+\gamma)(\mu+\alpha+\rho)}, \\
& D=1-\mathscr{R}_{P_{T}} .
\end{aligned}
$$

Solving for $\lambda^{*}$ in $h\left(\lambda^{*}\right)=0$, the roots of $h\left(\lambda^{*}\right)=0$ are explored using the Descartes's rule of signs. The various possibilities are tabulated in Table 1.

The analysis of the results in Table 1 results in the following Theorem 2.

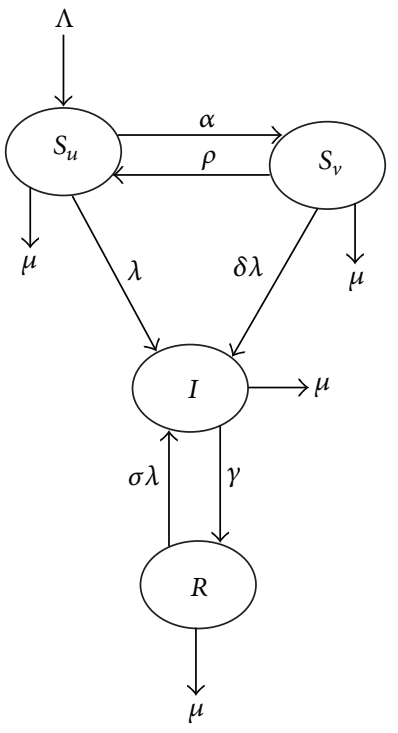

FIGURE 1: Structure of the model.

Theorem 2. The scabies model (1)

(i) has a unique endemic equilibrium if $\mathscr{R}_{P_{T}}>1$ and whenever Cases 1, 2, 3, and 4 are satisfied;

(ii) could have more than one endemic equilibrium if $\mathscr{R}_{P_{T}}>1$ and Case 2 is satisfied;

(iii) could have two endemic equilibria if $\mathscr{R}_{P_{T}}<1$ and cases 2, 3, and 4 are satisfied.

3.2. Analysis of the Reproduction Number $\mathscr{R}_{P_{T}}$. In the absence of both vaccination and treatment $\mathscr{R}_{P_{T}}$ is simply the basic reproduction number $\mathscr{R}_{0}$ which is defined as the number of secondary scabies cases produced by one infectious 


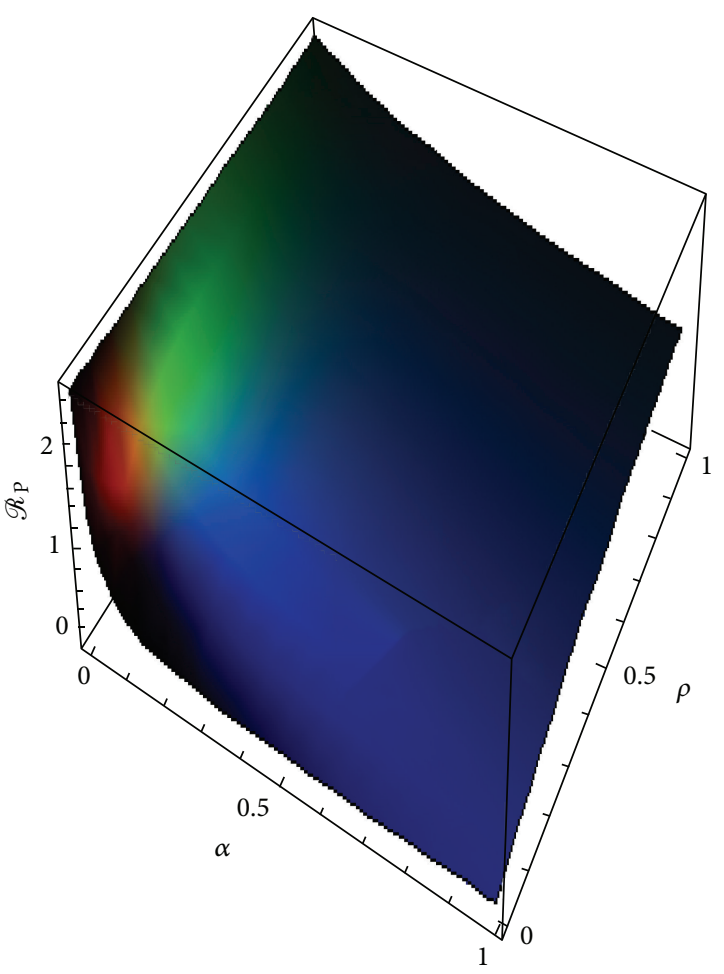

FIGURE 2: Effects of vaccination rate and vaccine waning rate on $\mathscr{R}_{P}$.

individual during his/her entire infectious period in the absence of any intervention strategy and is given by

$$
\mathscr{R}_{0}=\frac{\beta}{\mu} \text {. }
$$

Now let us analyse what happens when their vaccination is only in the community. In that scenario, $\mathscr{R}_{P_{T}}$ becomes $\mathscr{R}_{P}$ which is defined as the number of secondary scabies cases produced by one infected individual during his/her entire infectious period in presence of vaccination and is given by

$$
\lim _{\gamma \rightarrow 0} \mathscr{R}_{P_{T}}=\mathscr{R}_{P}=\frac{\beta(\mu+\rho+\delta \alpha)}{\mu(\mu+\rho+\alpha)}=\frac{\mu+\rho+\delta \alpha}{\mu+\rho+\alpha} \mathscr{R}_{0} .
$$

Subtracting $\mathscr{R}_{P}$ from $\mathscr{R}_{0}$, we have

$$
\Delta_{p}=\mathscr{R}_{P}-\mathscr{R}_{0}=\frac{(1-\delta) \alpha}{\mu+\rho+\alpha} \mathscr{R}_{0}>0 .
$$

Also, partially differentiating $\mathscr{R}_{P}$ with respect to $\alpha$, one gets

$$
\frac{\partial \mathscr{R}_{P}}{\partial \alpha}=-\frac{(\mu+\rho)(1-\delta)}{(\mu+\rho+\alpha)^{2}} \mathscr{R}_{0}<0 .
$$

The fact that (11) is positive and (12) is negative suggests that the increase in the use of the vaccine will increase its benefit to the community. This result suggests more resources should be geared towards the development and deployment of this possible vaccine against scabies. Now we determine the critical vaccination rate necessary for the vaccine to have a positive impact on its control and this is done by expressing $\alpha$ as a subject of the formula in (10) to obtain

$$
\alpha^{c}=\frac{(\mu+\rho)\left(1-\mathscr{R}_{P} / \mathscr{R}_{0}\right)}{\mathscr{R}_{P} / \mathscr{R}_{0}-\delta} \text { and exists for } \delta<\frac{\mathscr{R}_{P}}{\mathscr{R}_{0}}<1 .
$$

Thus, as long as $\alpha>\alpha^{c}$, then vaccination will aid in the control of scabies. However, for $\alpha<\alpha^{c}$, vaccination levels will not be high enough to make a meaningful impact on scabies control. To effectively determine the effects vaccination has on scabies control, there is a need to know the critical vaccine waning rate which is found by making $\rho$ the subject of formula in the equation of $\mathscr{R}_{P}$ to obtain

$$
\begin{gathered}
\rho^{c}=\frac{(\mu+\delta \alpha)\left(\left((\mu+\alpha) \mathscr{R}_{P} /(\mu+\delta \alpha) \mathscr{R}_{0}\right)-1\right)}{1-\mathscr{R}_{P} / \mathscr{R}_{0}} \\
\text { which exists for } \frac{\mu+\delta \alpha}{\mu+\alpha}<\frac{\mathscr{R}_{P}}{\mathscr{R}_{0}}<1 .
\end{gathered}
$$

Thus, vaccination will be able to control scabies provided $\rho<\rho^{c}$. If $\rho>\rho^{c}$, then the rate at which the vaccine wanes is very high that herd immunity cannot be achieved. Table 2 summarises the effects of vaccination and vaccine waning rates.

Results from Table 2 show that an ideal situation for a vaccine to achieve herd immunity is when vaccination coverage rates are high and vaccine waning rates are low $\left(\alpha>\alpha^{c}, \rho<\rho^{c}\right)$. This result is further illustrated in Figure 2 which shows that high vaccination rates accompanied by low vaccine waning rates are ideal to make the reproduction number $\mathscr{R}_{P}$ small. A reduction in the size of the reproduction number translates to a reduction in the number of new infections.

Since we are talking about vaccination and its potential impact of scabies control, there is a need to find the critical protective factor for a vaccine to be effective. This requires making $\delta$ the subject of the formulae in $\mathscr{R}_{P}$ and doing so one gets

$$
\delta^{c}=\frac{\mu+\rho}{\alpha}\left(\frac{(\mu+\rho+\alpha) \mathscr{R}_{P}}{(\mu+\rho) \mathscr{R}_{0}}-1\right)
$$

$$
\text { which exists for } \frac{\mu+\rho}{\mu+\rho+\alpha}<\frac{\mathscr{R}_{P}}{\mathscr{R}_{0}}<1 \text {. }
$$

If $\delta<\delta^{c}$, then the vaccine will be effective enough to control scabies infection. However, when $\delta>\delta^{c}$, then vaccination will not contribute meaningfully to the reduction of scabies. Looking at the nature of $\mathscr{R}_{P_{T}}$ shows that it is a decreasing function of $\gamma$ suggesting the benefits the community gets by increasing levels of treatment. Is vaccination necessary in a community where treatment is there? To answer this question, we partially differentiate $\mathscr{R}_{P_{T}}$ with respect to $\alpha$ and we obtain

$$
\frac{\partial \mathscr{R}_{P_{T}}}{\partial \alpha}=-\frac{\mu \mathscr{R}_{0}(\mu+\rho)(1-\delta)}{(\mu+\gamma)(\mu+\rho+\alpha)^{2}}<0 .
$$




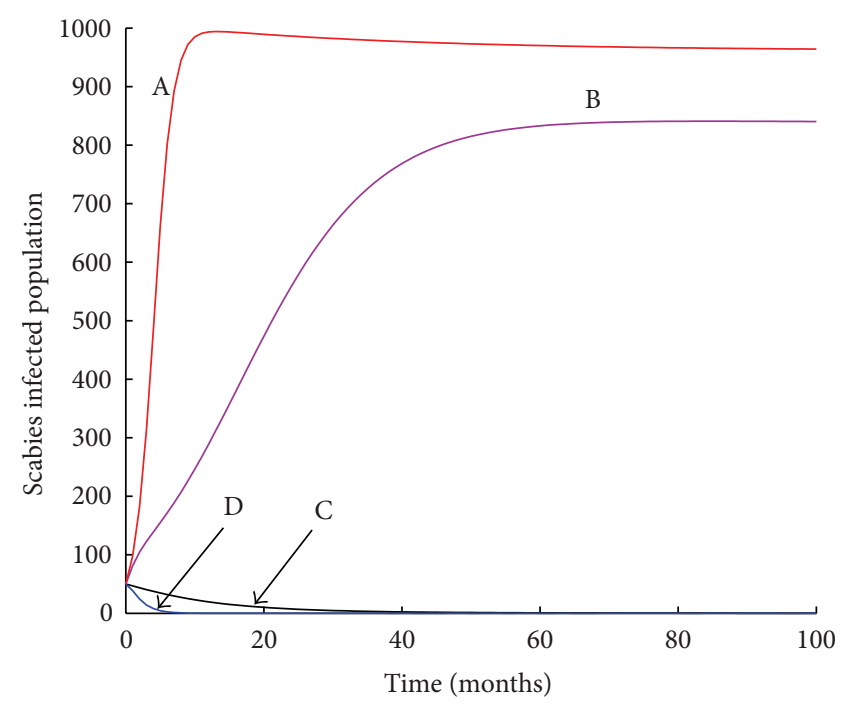

(a)

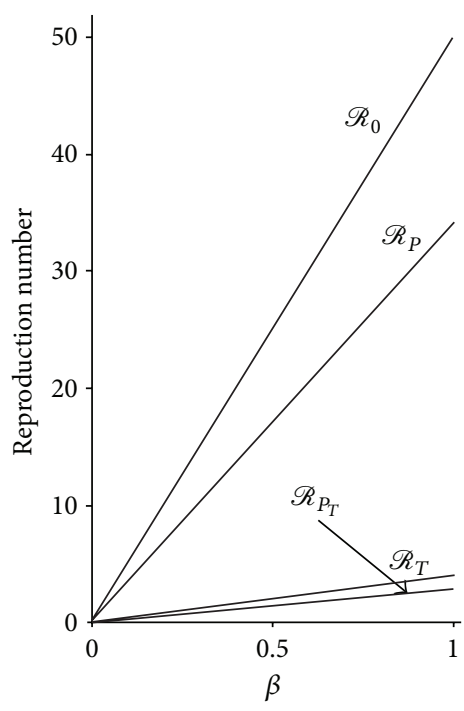

(b)

FIGURE 3: Effects of vaccination and treatment. A denotes when there is no intervention, B denotes when vaccination is the only intervention strategy to fight scabies, $\mathrm{C}$ denotes when scabies treatment is the only intervention, and $\mathrm{D}$ denotes when vaccination and treatment are both used to control scabies. Parameter values used are in Table 3.

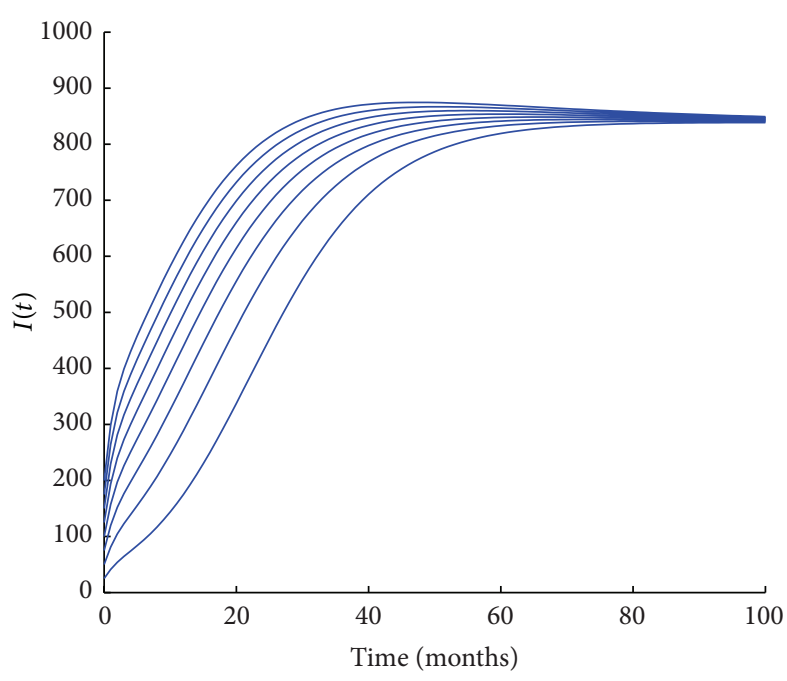

(a)

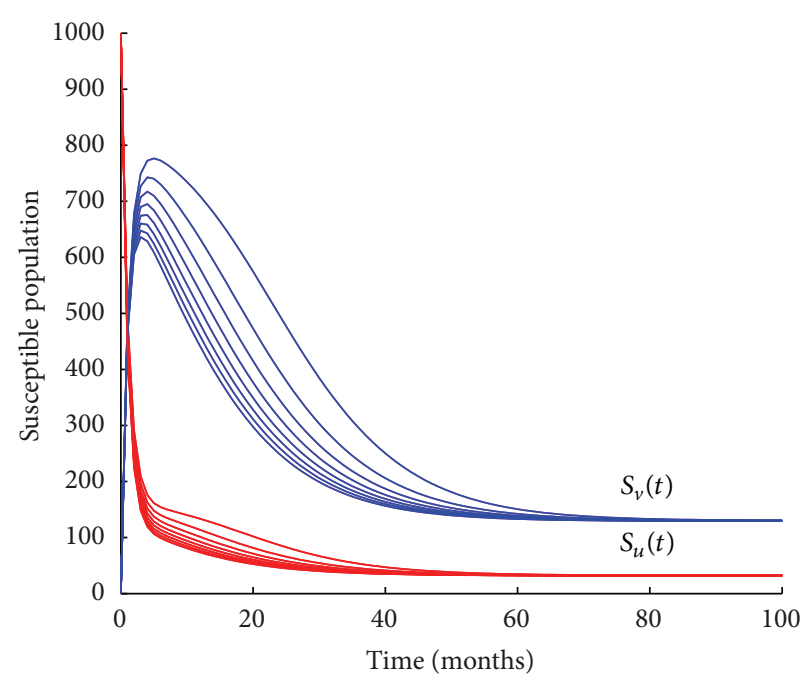

(b)

FIGURE 4: Simulations of model system (1) showing plots of the susceptibles and scabies infected as a function of time with various initial conditions when $\mathscr{R}_{P}>1\left(\mathscr{R}_{P}=1.545\right)$. Parameter values used are in Table 3 .

The fact that (16) is negative suggests that even in communities where treatment of scabies is already available the community will also benefit scabies vaccination.

\section{Numerical Simulations}

In this section, we carry out detailed numerical simulations using MATLAB programming language to assess the possible effects intervention strategies will have on scabies control. The parameter values that we use for numerical simulations are in Table 3. In Table 3, CSOZ denotes Central Statistical Office of Zimbabwe. For vaccination and vaccine waning rates, we used estimates used in possible $\mathrm{TB}$ vaccines by Bhunu et al. [5], just for illustration purposes.
Figure 3 shows the effect of vaccination and treatment on the actual scabies cases and on the reproduction numbers obtained for different scenarios. This in a way allows comparison of vaccination and treatment as intervention strategies to control scabies. Figure 3(a) is graphical representation of scabies infected population under different intervention strategies. It shows that in the absence of any intervention strategy the scabies infected population increases from the onset and reaches a state where it remains constant. In the presence of vaccination, only the number of scabies increases from the onset until it reaches its asymptotic state where it remains more or less constant. It is worth mentioning here that trend $\mathrm{A}$ is always greater than trend $\mathrm{B}$ apart from 


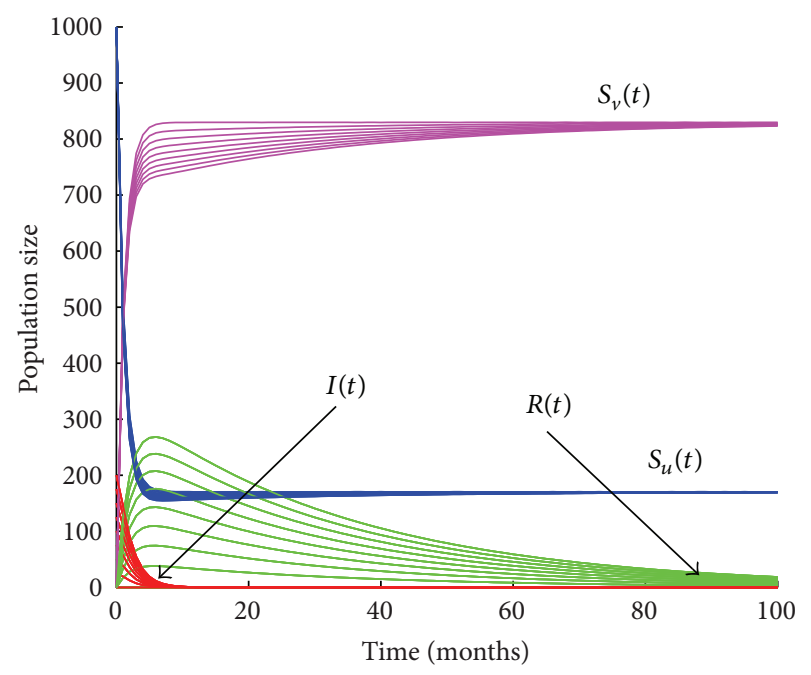

Figure 5: Simulations of model system (1) showing plots of the susceptibles and scabies infected as a function of time for various initial conditions when $\mathscr{R}_{P_{T}}<1\left(\mathscr{R}_{P_{T}}=0.875\right)$. Parameter values used are in Table 3.

the starting point. This suggests that vaccination will partially reduce scabies infection. However, the situation is different when treatment is involved as the only intervention strategy, and the number of scabies case declines to asymptotically low levels, suggesting that treatment alone can control scabies infections. In the case where vaccination of susceptibles is coupled with treatment infectives, the scabies infected population falls off more rapidly to asymptotically low levels than when treatment is used as the only intervention strategy. Vaccination reduces the rate at which individuals are infected and treatment kills off scabies causing mites. Figure 3(b) illustrates relationship and behavior of $\mathscr{R}_{0}, \mathscr{R}_{P}, \mathscr{R}_{T}, \mathscr{R}_{P_{T}}$ for varying rate of acquiring scabies infection. It shows that $\mathscr{R}_{0}>$ $\mathscr{R}_{P}>\mathscr{R}_{T}>\mathscr{R}_{P_{T}}$ for the given parameter values and when there is one scabies infected individual in a pool of exclusively susceptible individuals. This implies that vaccination coupled with treatment is the most effective way of combating scabies in a community. Comparing results in Figure 3(a) with those in Figure 3(b), we conclude that vaccination coupled with treatment is the best approach to control scabies as evidenced by the depletion of scabies infected cases in Figure 3(a) and a very small reproduction number in Figure 3(a). By looking at trends $\mathrm{C}$ and D (Figure $3(\mathrm{a})$ ) and $\mathscr{R}_{T}$ and $\mathscr{R}_{P_{T}}$ (Figure $3(\mathrm{~b})$ ), we safely also conclude that treatment alone is also effective in scabies control.

In Figure 4, effects of varying initial conditions when the vaccination induced reproduction is greater than unity are illustrated. It shows that the susceptibles and scabies infected converge to their corresponding endemic equilibrium states. It is worth mentioning that there is no recovered population in this case as once an individual has been infected, he/she remains so in the absence of treatment.

Figure 5 graphically shows what happens when $\mathscr{R}_{P_{T}}<$ 1 for various initial conditions. It shows that all the sub-populations of people converge to their respective disease-free equilibrium states. This signifies that making the reproduction number less than unity is necessary for disease eradication.

\section{Discussion}

A mathematical model for the transmission dynamics of scabies has been proposed and investigated. The potential of a possible preexposure vaccine and treatment have been analysed and compared in an effort to understand the best possible scabies control strategies. The disease-free equilibrium is shown to be locally asymptotically stable when the corresponding reproduction number is less than unity. The possible endemic equilibria states of the model have been explored using the Descartes' rule of signs. Analysis of the reproduction number has shown that vaccination has some potential to control the scabies epidemic. Additionally, it has been shown that, in communities where scabies treatment is available, adding vaccination into that community will increase the potential to control scabies. It has been shown that to effectively control scabies through vaccination alone there should be high vaccination coverage rates with a vaccine which does not wane too quickly. Numerical simulations also support the analytical results obtained that is vaccination coupled with treatment is the best possible approach to successfully fight scabies in a given community. Furthermore, numerical simulations show that treatment alone is able to control scabies as noted by the depletion of scabies cases and low reproduction number when it is used as the only intervention strategy. This is good news considering that currently there is no preexposure vaccine against scabies. However, given the fact that the most effective medications for scabies are beyond the reach of many, a development and deployment of a "new" vaccine will help in controlling the spread of scabies especially among the disadvantaged communities.

\section{References}

[1] J. S. McCarthy, D. J. Kemp, S. F. Walton, and B. J. Currie, "Scabies: more than just an irritation," Postgraduate Medical Journal, vol. 80, no. 945, pp. 382-387, 2004.

[2] J. M. Tielsch and A. Beeche, "Impact of ivermectin on illness and disability associated with onchocerciasis," Tropical Medicine and International Health, vol. 9, no. 4, pp. A45-A56, 2004.

[3] K. Mellanby, "Scabies in 1976," Royal Society of Health Journal, vol. 97, no. 1, pp. 32-40, 1977.

[4] D. Landwehr, S. M. Keita, J. M. Pönnighaus, and C. Tounkara, "Epidemiologic aspects of scabies in Mali, Malawi, and Cambodia," International Journal of Dermatology, vol. 37, no. 8, pp. 588-590, 1998.

[5] C. P. Bhunu, W. Garira, Z. Mukandavire, and G. Magombedze, "Modelling the effects of pre-exposure and post-exposure vaccines in tuberculosis control," Journal of Theoretical Biology, vol. 254, no. 3, pp. 633-649, 2008.

[6] D. Taplin, T. L. Meinking, J. A. Chen, and R. Sanchez, "Comparison of crotamiton $10 \%$ cream (Eurax) and permethrin $5 \%$ cream (Elimite) for the treatment of scabies in children," Pediatric Dermatology, vol. 7, no. 1, pp. 67-73, 1990.

[7] A. J. O. Scabies, Arthropods and Human Skin, Springer, New York, NY, USA, 1984. 
[8] A. M. H. Gray, "Treatment of scabies" British Journal of Dermatology and Syphilis, vol. 53, p. 148, 1941.

[9] J. A. van den Hoek, J. A. van de Weerd, T. D. Baayen et al., "A persistent problem with scabies in and outside a nursing home in Amsterdam: indications for resistance to lindane and ivermectin," Euro Surveillance, vol. 13, no. 48, pp. 5-6, 2008.

[10] K. Mellanby, "The development of symptoms, parasitic infection and immunity in human scabies," Parasitology, vol. 35, pp. 197-206, 1944.

[11] L. G. Arlian, C. M. Rapp, and M. S. Morgan, "Resistance and immune response in scabies-infested hosts immunized with Dermatophagoides mites," American Journal of Tropical Medicine and Hygiene, vol. 52, no. 6, pp. 539-545, 1995.

[12] L. G. Arlian, M. S. Morgan, D. L. Vyszenski-Moher, and B. L. Stemmer, "Sarcoptes scabiei: the circulating antibody responses and induced immunity to scabies," Experimental Parasitology, vol. 78, no. 1, pp. 37-50, 1994.

[13] A. B. Shrank and S. L. Alexander, "Scabies: another epidemic?" British Medical Journal, vol. 1, no. 541, pp. 669-671, 1967.

[14] P. van den Driessche and J. Watmough, "Reproduction numbers and sub-threshold endemic equilibria for compartmental models of disease transmission," Mathematical Biosciences, vol. 180, pp. 29-48, 2002. 

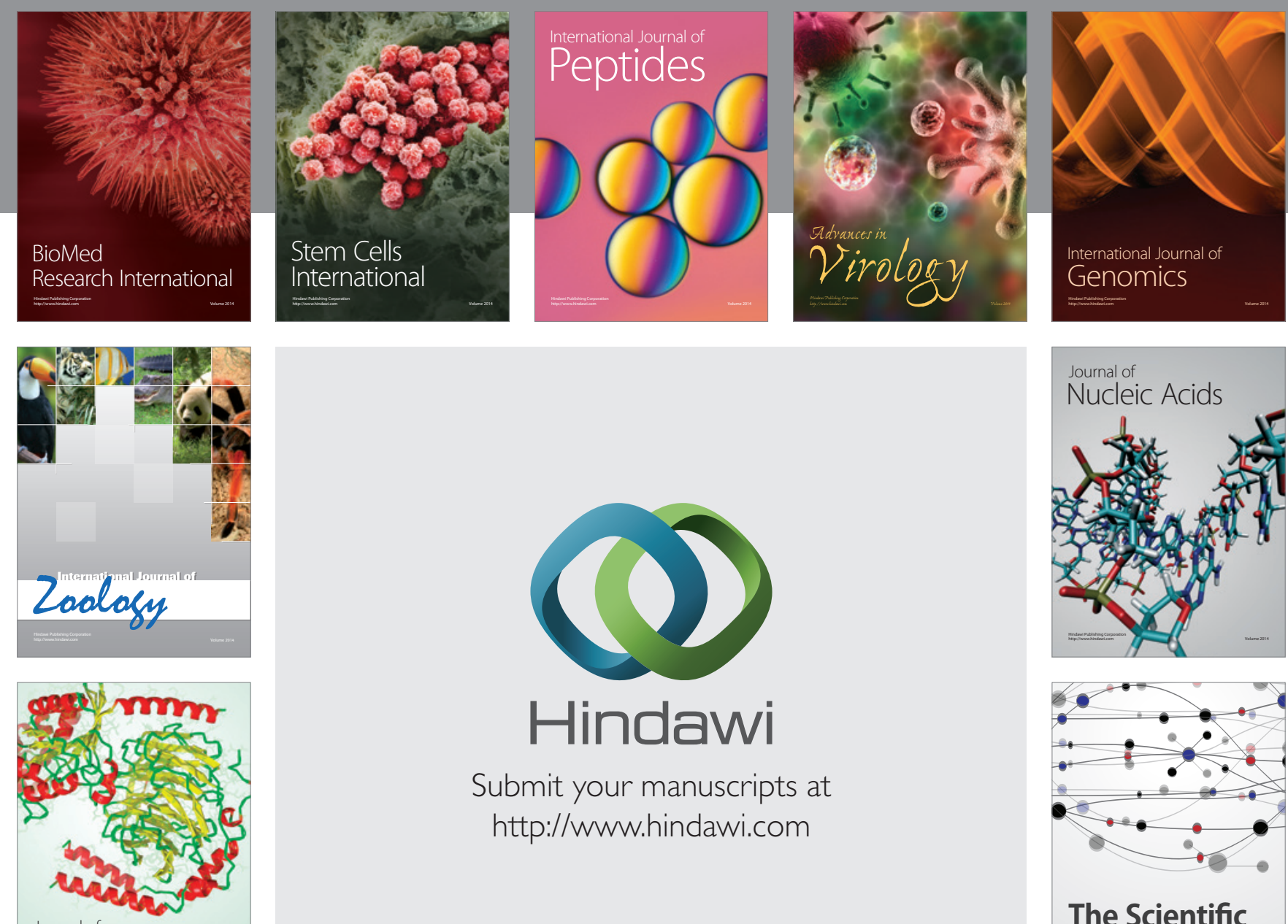

Submit your manuscripts at

http://www.hindawi.com

Journal of
Signal Transduction
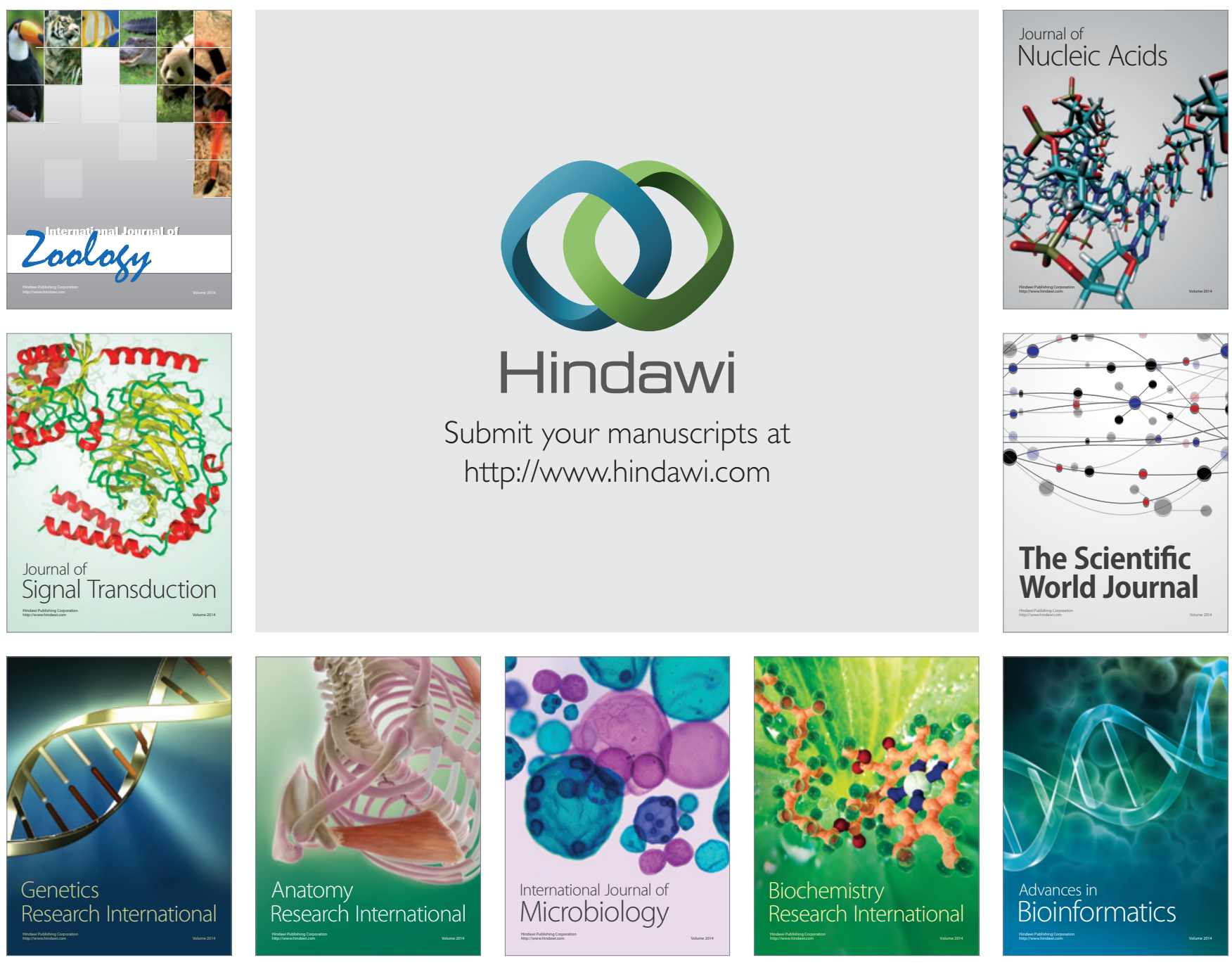

The Scientific World Journal
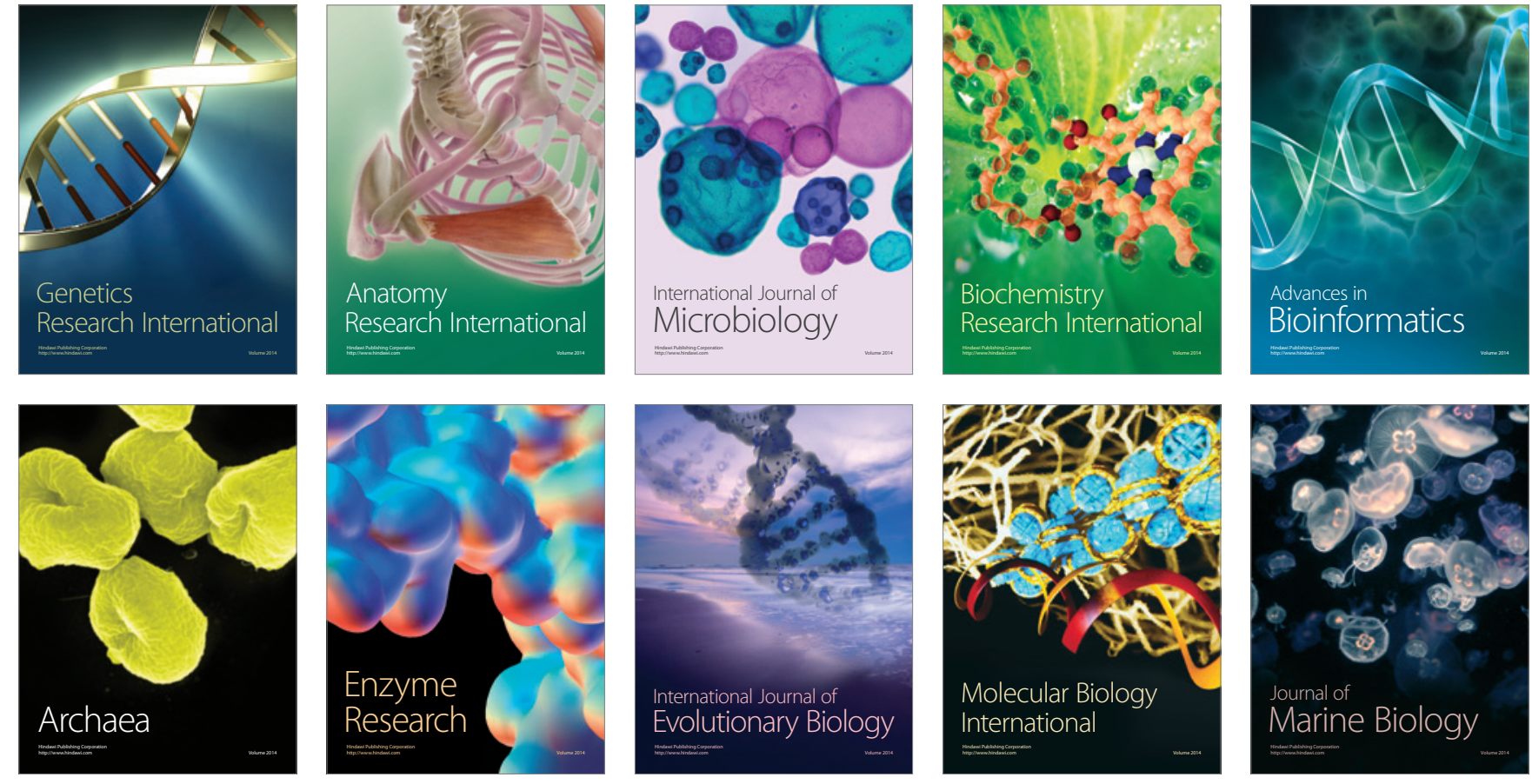